\title{
Spectroscopic comparison of aqueous altered asteroids with CM2 carbonaceous chondrite meteorites ${ }^{\star}$
}

\author{
S. Fornasier ${ }^{1}$, M. Lazzarin $^{1}$, C. Barbieri ${ }^{1}$, and M.A. Barucci ${ }^{2}$ \\ 1 Dip. di Astronomia, Vic. Osservatorio 5, I-35122 Padova, Italy \\ e-mail: fornasier@pd.astro.it, lazzarin@pd.astro.it, barbieri@pd.astro.it \\ 2 Observatoire de Paris, 92195 Meudon Principal Cedex, France \\ e-mail: barucci@obspm.fr
}

Received July 15; accepted September 23, 1998

\begin{abstract}
In the last year we have started a spectroscopic investigation of asteroids located in the region of the mainbelt between about 2.2 and 3.6 AU. The aim of this work is to study the aqueous alteration process which acted in that zone, dominated by low albedo C-type asteroids, and to compare the spectra of these hydrous objects with those of CM2 carbonaceous chondrite meteorites. In fact, the spectra of these meteorites reveal features probably due to aqueous altered materials on their surfaces.

The study of the aqueous alteration process can give important information on the chemical and thermal evolution of the earliest Solar System.

More that $65 \%$ of the investigated objects have revealed features suggesting the presence of hydrous materials. The comparison of the spectra of the hydrated asteroids obtained to date with those of several CM2 carbonaceous chondrite meteorites seems to indicate that aqueous altered asteroids could be the parents of CM2 meteorites.

The data have been obtained during several observational runs at the Asiago Observatory with the $1.8 \mathrm{~m}$ telescope and at ESO-LaSilla with the $1.5 \mathrm{~m}$ telescope.
\end{abstract}

Key words: meteors, meteoroids - minor planets, asteroids - solar systems: formation

\section{Introduction}

The origin of most meteorite types is still a matter of debate (asteroidal, cometary or planetary origin).

Send offprint requests to: S. Fornasier

* Based on observations carried out at the European Southern Observatory (ESO), La Silla, Chile, and at the Asiago Observatory, Italy.
In this work we attempted a comparison between hydrated asteroids and CM2 carbonaceous chondrites as they exhibit similar spectroscopic behaviour.

The distribution of asteroidal classes is dependent on heliocentric distance: objects closer to the Sun (belonging above all to S-type), appear to have been strongly heated and differentiated, while asteroids at greater distance seem to have undergone little or no heating and differentiation. These ones belong to $\mathrm{C}, \mathrm{P}$ and $\mathrm{D}$ taxonomic types: they are low-albedo $(\leq 0.05)$ objects, darkened by carbonaceous and organic materials on their surfaces (Gradie \& Tedesco 1982). So the small bodies at great heliocentric distances $\left(d_{\odot} \geq 3 \mathrm{AU}\right)$ may have preserved materials which witnessed the condensation of the Solar nebula and the early phases of the formation of the Solar System. Vilas (1994), has revealed that a particular zone of the outer main belt seems dominated by objects which have undergone aqueous alteration process, that is the low temperature chemical alteration of materials by liquid water which acts as a solvent and produces materials like phyllosilicates, sulfates, oxides, carbonates, and hydroxides. The study of this process can give important information on the evolution of the earliest Solar System. It is now believed that hydrous minerals could not have condensed directly from the solar nebula, but that they have been produced by hydration of pristine anhydrous silicates. This means that water was present in the primordial asteroids, probably in the form of ice condensed together with the lithic material. Successively, an heat source, probably electric induction by the solar wind during the T-Tauri phase of our Sun (as suggested by Shimazu \& Teresawa 1995; Herbert 1989) melted the ice and produced the liquid water necessary to alter superficial minerals. As liquid water can exist only under particular conditions of temperature and pressure, the study of aqueous alteration processes can help to reconstruct the 
different evolutionary stages of the chemical and thermal history of our Solar System.

The asteroids that show hydrated minerals on their surfaces seem to dominate the region of the main belt between 2.6 and 3.5 AU, called also "aqueous alteration zone" (Vilas 1994), and belong essentially to the C, G, F and $\mathrm{B}$ taxonomic classes.

Hydrated materials produce characteristic absorption features on the spectra of the asteroids: in the infrared region the $3 \mu \mathrm{m}$ band (Lebofsky 1980; Jones et al. 1990), associated to "free" water molecules, and to $\mathrm{OH}$ ion bounded in the mineral crystal lattice; in the visible range there are several bands centered around $0.43 \mu \mathrm{m}, 0.60-0.65 \mu \mathrm{m}$, $0.70 \mu \mathrm{m}$ and $0.80-0.90 \mu \mathrm{m}$, attributed to $\mathrm{Fe}^{2+} \rightarrow \mathrm{Fe}^{3+}$ charge transfer transitions in oxidized iron (Vilas et al. 1993, 1994; Vilas 1994; Barucci et al. 1998).

In this context we have started a spectroscopic survey of asteroids located between about 2 and 4 AU. Moreover, we attempted a comparison of the spectra of hydrated asteroids with those of CM2 carbonaceous chondrites which show features due to aqueous altered materials, in order to obtain information about the origin of $\mathrm{CM} 2$ chondrites.

\section{Observations and data reduction}

The data of the asteroids presented have been obtained during different observing runs in the course of 1997.

The observations were performed at the European Southern Observatory of La Silla (Chile) and at the Asiago Astrophysical Observatory (Italy).

At La Silla we used the $1.5 \mathrm{~m}$ telescope equipped with a Boller \& Chivens spectrograph and a Loral Lesser CCD as detector $(2048 \times 2048$ pixels $)$. The grating used was a $225 \mathrm{gr} / \mathrm{mm}$, with a dispersion of $331 \AA / \mathrm{mm}$ in the first order. The CCD has a $15 \mu \mathrm{m}$ square pixels, giving a dispersion of about $5 \AA$ /pixel in the wavelength direction. The spectral range is about $0.48<\lambda<0.92 \mu \mathrm{m}$ with a FWHM of about $10 \AA$.

At the Asiago Observatory we used the $1.82 \mathrm{~m}$ telescope equipped with a Boller \& Chivens spectrograph and a Thomson CCD $(430 \times 600$ pixels $)$ as detector. The grating was a $150 \mathrm{gr} / \mathrm{mm}$ with a dispersion of $340 \AA / \mathrm{mm}$ in the first order. The CCD has $23 \mu \mathrm{m}$ square pixels giving a dispersion of about $7.8 \AA$ /pixel in the wavelength direction. The spectral coverage is about $0.5<\lambda<0.9 \mu \mathrm{m}$ with an instrumental FWHM of $15.6 \AA$.

Each spectrum was recorded through a slit oriented in the East-West direction. The slit was opened to about 8 arcsec in order to reduce effects due to differential refraction and the possibility of losing signal due to guiding errors of the telescopes.

In Table 1 we report the circumstances of the observations (date and site), the visual magnitude of the asteroids, the solar analog stars used for reduction and some physical characteristics of the observed objects: semimajor

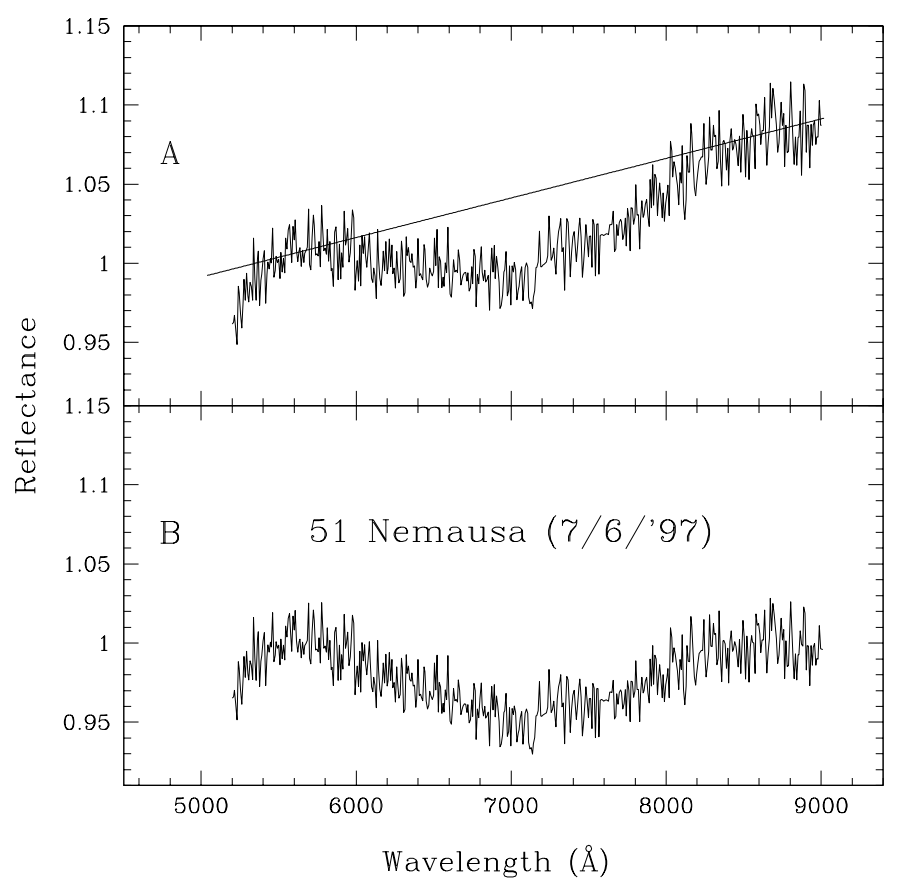

Fig. 1. a) reflectance spectrum of the asteroid 51 Nemausa with superimposed its linear continuum, computed with a linear least squares fit to the smoothed spectral data points. b) residual spectra of 51 Nemausa created as a result of the asteroid spectra in A being divided by the linear background. The same procedure was applied to all the other asteroids

axis (AU), diameter $(\mathrm{km})$ and albedo derived from IRAS observations, and taxonomic type (Tholen taxonomy).

During each night, we also recorded bias, flat-field, calibration lamp, spectrophotometric standard and solar analog stars spectra at different intervals throughout the night. The stars were observed at airmasses similar to those of the objects.

Solar analog stars (Hardorp 1978) are fundamental in the final step of the reduction procedure to remove the solar contribution from the spectra of the asteroids and to obtain the asteroidal reflectivities. Eight stars have been used: Hyades64, 16 Cyg B, HD 28022, HD 44594, HD 89010, HD 20630, HD 86728, HD 76151. Their choice is connected with the observational period.

The spectra were reduced using ordinary procedures of data reduction with the software packages Midas and IDL.

These procedures include: subtraction of the bias from the raw data, flattening of the data in order to remove large scale structures, cosmic ray removal, background subtraction, collapsing the two dimensional spectra, wavelength calibration, atmospheric extinction. The reflectivity of the asteroids was then obtained by dividing the spectra of the objects by the respective solar analog spectrum.

All asteroid spectra are normalized at 1 around $5500 \AA$.

Spectra used in these studies, even with a good signal to noise ratio, have been smoothed with a median 
Table 1. Observational and physical characteristics of the observed asteroids: circumstances of the observations (date and site), visual magnitude of the asteroids, the solar analog stars used for reduction, semimajor axis (AU), diameter (km) and albedo derived from IRAS observations, and taxonomic type (Tholen taxonomy)

\begin{tabular}{|c|c|c|c|c|c|c|c|c|}
\hline Asteroid & Date & Site & $m_{v}$ & Solar An. & $a(\mathbf{A U})$ & $D(\mathbf{K m})$ & Albedo & $\mathbf{T}$ \\
\hline 1 Ceres & $12 / 11 / ' 97$ & $\mathrm{ESO}$ & 9.0 & HD28022 & 2.76 & 932 & 0.100 & G \\
\hline 10 Hygiea & 12/13/'97 & $\mathrm{ESO}$ & 10.2 & HD44594 & 3.13 & 429 & 0.075 & $\mathrm{C}$ \\
\hline 19 Fortuna & 12/12/'97 & $\mathrm{ESO}$ & 11.5 & Hyades64 & 2.44 & 192 & - & G \\
\hline 19 Fortuna & 9/3/'97 & Asiago & 9.7 & 16 Cyg B & 2.44 & 192 & - & G \\
\hline 24 Themis & $5 / 3 / ' 97$ & Asiago & 12.2 & HD 89010 & 3.12 & 234 & - & $\mathrm{C}$ \\
\hline 34 Circe & $9 / 3 / ' 97$ & Asiago & 13.1 & 16 Cyg B & 2.68 & 118 & 0.054 & $\mathrm{C}$ \\
\hline 38 Leda & $5 / 3 / ' 97$ & Asiago & 12.8 & HD 89010 & 2.74 & 120 & 0.062 & $\mathrm{C}$ \\
\hline 41 Daphne & 12/13/'97 & ESO & 11.9 & HD20630 & 2.76 & 182 & 0.073 & $\mathrm{C}$ \\
\hline 45 Eugenia & 9/4/'97 & Asiago & 11.3 & 16 Cyg B & 2.72 & 214 & 0.040 & $\mathrm{C}$ \\
\hline 51 Nemausa & $7 / 6 / ' 97$ & Asiago & 10.5 & 16 Cyg B & 2.36 & 153 & 0.092 & $\mathrm{C}$ \\
\hline 51 Nemausa & 9/3/'97 & Asiago & 11.7 & 16 Cyg B & 2.36 & 153 & 0.092 & $\mathrm{C}$ \\
\hline 65 Cybele & 12/13/'97 & $\mathrm{ESO}$ & 12.5 & HD20630 & 3.42 & 230 & 0.057 & $\mathrm{P}$ \\
\hline 70 Panopaea & 12/12/'97 & $\mathrm{ESO}$ & 12.8 & Hyades64 & 2.61 & 127 & 0.070 & $\mathrm{C}$ \\
\hline 74 Galatea & 12/13/'97 & $\mathrm{ESO}$ & 12.2 & Hyades64 & 2.78 & 123 & 0.034 & $\mathrm{C}$ \\
\hline 104 Klymene & 12/13/'97 & $\mathrm{ESO}$ & 12.6 & Hyades64 & 3.16 & 127 & 0.052 & $\mathrm{C}$ \\
\hline 105 Artemis & 12/13/'97 & ESO & 12.9 & Hyades64 & 2.37 & 123 & 0.032 & $\mathrm{C}$ \\
\hline 128 Nemesis & $5 / 2 / ' 97$ & Asiago & 12.9 & HD86728 & 3.09 & 194 & 0.050 & $\mathrm{C}$ \\
\hline 130 Elektra & 9/4/'97 & Asiago & 10.4 & 16 Cyg B & 3.11 & 189 & 0.076 & G \\
\hline 137 Meliboea & 12/13/'97 & ESO & 13.1 & Hyades64 & 3.11 & 150 & 0.048 & $\mathrm{C}$ \\
\hline 144 Vibilia & 12/13/'97 & ESO & 11.0 & HD20630 & 2.65 & 146 & 0.059 & $\mathrm{C}$ \\
\hline 145 Adeona & $5 / 3 / ' 97$ & Asiago & 11.9 & 16 Cyg B & 2.67 & 155 & 0.043 & $\mathrm{C}$ \\
\hline 146 Lucina & 12/13/'97 & ESO & 13.6 & Hyades64 & 2.71 & 137 & 0.052 & $\mathrm{C}$ \\
\hline 185 Eunike & 5/3/'97 & Asiago & 12.4 & 16 Cyg B & 2.73 & 165 & 0.064 & $\mathrm{C}$ \\
\hline 190 Ismene & 12/13/'97 & ESO & 12.7 & Hyades64 & 3.97 & 212 & - & $\mathrm{P}$ \\
\hline 200 Dynamene & 12/13/'97 & ESO & 13.1 & Hyades64 & 2.73 & 132 & 0.053 & $\mathrm{C}$ \\
\hline 211 Isolda & $5 / 2 / ’ 97$ & Asiago & 13.4 & HD76151 & 3.04 & 148 & 0.060 & $\mathrm{C}$ \\
\hline 238 Hypatia & $5 / 4 / ' 97$ & Asiago & 13.0 & 16 Cyg B & 2.90 & 156 & 0.043 & $\mathrm{C}$ \\
\hline 304 Olga & 12/13/'97 & ESO & 13.4 & Hyades64 & 2.40 & 68 & 0.047 & $\mathrm{C}$ \\
\hline 410 Chloris & 12/13/'97 & ESO & 13.5 & Hyades64 & 2.72 & 128 & 0.054 & $\mathrm{C}$ \\
\hline 444 Gyptis & 7/7/'97 & Asiago & 11.2 & 16 Cyg B & 2.76 & 170 & 0.051 & $\mathrm{C}$ \\
\hline 488 Kreusa & $5 / 3 / ' 97$ & ESO & 11.8 & HD86728 & 3.14 & 158 & 0.059 & $\mathrm{C}$ \\
\hline 490 Veritas & 7/7/'97 & Asiago & 13.5 & 16 Cyg B & 3.16 & 121 & 0.062 & $\mathrm{C}$ \\
\hline 618 Elfreide & $12 / 13 / ' 97$ & $\mathrm{ESO}$ & 13.6 & Hyades64 & 3.18 & 124 & 0.058 & $\mathrm{C}$ \\
\hline 712 Boliviana & 12/13/'97 & $\mathrm{ESO}$ & 11.8 & Hyades64 & 2.57 & 132 & 0.046 & $\mathrm{C}$ \\
\hline 776 Berbericia & 12/13/'97 & $\mathrm{ESO}$ & 12.4 & Hyades64 & 2.93 & 178 & - & $\mathrm{C}$ \\
\hline 1093 Freda & $5 / 1 / ' 97$ & Asiago & 13.3 & HD89010 & 3.13 & 120 & 0.038 & $\mathrm{C}$ \\
\hline
\end{tabular}

filter technique. Moreover, in order to study aqueous absorption features, which may be very weak, we treated each asteroid/solar-analog-spectrum as a continuum with discrete absorption features superimposed on it, as described by Vilas et al. (1993, 1993b). For the spectra, a simple linear continuum is defined by a linear least squares fit to the smoothed spectral data points. We then divided each individual spectrum by the continuum, thus removing a sloped background (Fig. 1). If residual absorption features are present in these processed spectra, these features can then be easily recognized. In Figs. 2 and 3 we report the spectra of the observed asteroids divided by their linear backgrounds.

\section{Results}

\subsection{Survey of asteroids}

Our spectroscopic survey reveals that more than $65 \%$ of the 34 observed asteroids show the presence of absorption bands due to aqueous alteration products, in particular of the $0.7 \mu \mathrm{m}$ band, which is the most characteristic feature of the hydrated materials in the visible.

The depth of these bands varies between $2 \%$ and $6 \%$ with respect to the continuum.

We considered only the absorption features deeper than the peak-to-peak scatter (that is $\leq 0.02$ ) in the spectrum, which, from previous experience, seems to be a better indicator of the spectrum quality than the calculated signal to noise ratio (Vilas \& Smith 1985). 


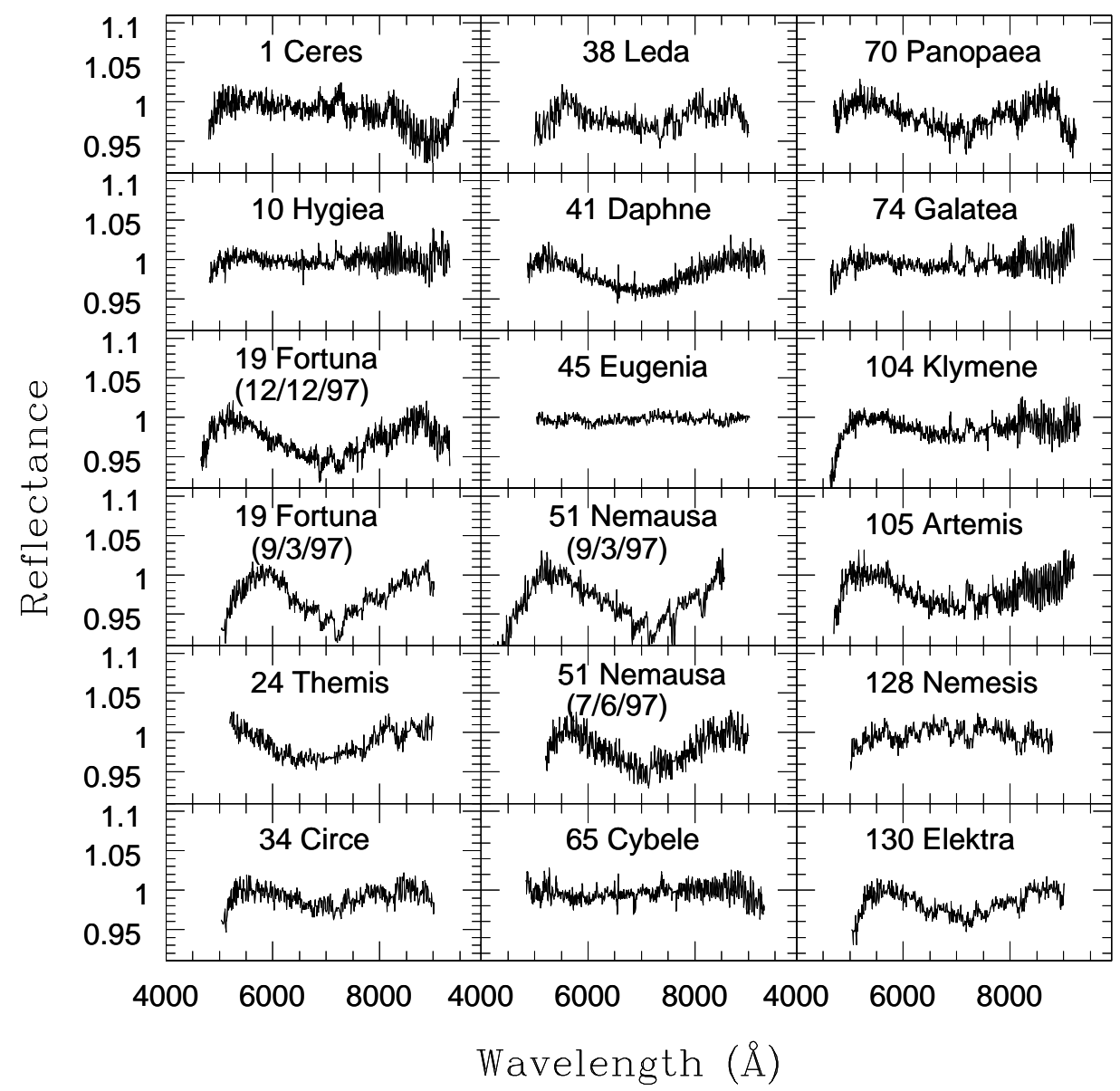

Fig. 2. Reflectance spectra of the observed asteroids. The spectra are normalized to $5500 \AA$ and a linear continuum has been removed

The repeatability of the $0.7 \mu \mathrm{m}$ absorption band in 19 Fortuna and 51 Nemausa, which were both observed twice on different observing runs, is a good indicator of the quality of data reduction.

Moreover the location and the extension of aqueous altered absorption characteristics do not match any atmospheric absorption band or solar analog feature.

The intense telluric water absorption beginning near $0.9 \mu \mathrm{m}$ coupled with a drop in responsivity of the CCD detectors have affected the identification of $0.8-0.9 \mu \mathrm{m}$ features in the asteroid spectra, which we have clearly identified only on 1 Ceres.

Some spectra present spurious features due to an incomplete removal of telluric $\mathrm{H}_{2} \mathrm{O}$ at 7300 and $8200 \AA$ and/or to the atmospheric $\mathrm{O}_{2} \mathrm{~A}$ and $\mathrm{O}_{2} \mathrm{~B}$ bands at 7619 and $6882 \AA$ respectively, but they do not influence the identification of aqueous altered bands.

Of six investigated objects located outside the "aqueous alteration zone", four have shown the presence of hydration features (Fig. 4). We think that more observations could help to understand the efficiency zone of the aqueous alteration process.
Our results are consistent with those obtained by Barucci et al. (1998), who found that more than $65 \%$ of their observed asteroids are hydrated. They also observed hydration features on asteroids located closer to the Sun than 2.6 AU.

Our data also confirm the existence of a relationship between the albedo of the objects and the aqueous alteration process (Fig. 5): the percentage of the observed hydrated asteroids grows as albedo increases. This relation may be explained with the progressive leaching of iron from silicates as the aqueous alteration proceeds. Leached iron (iron is the most important opaque phase in the visible range associated to aqueous alteration process) would be enveloped into magnetite and iron sulfide grains, so less material would be available to absorb the incoming sunlight and this would cause the increasing of the albedo (Vilas 1994).

All the 3 G-class observed asteroids (1 Ceres, 19 Fortuna, 130 Elektra) have features attributed to aqueous altered materials, confirming the fact that G-type objects seem to be the most aqueous altered asteroids. In fact aqueous alteration sequence seems to begin with $\mathrm{P}$ class objects (the least altered) and to increase through 


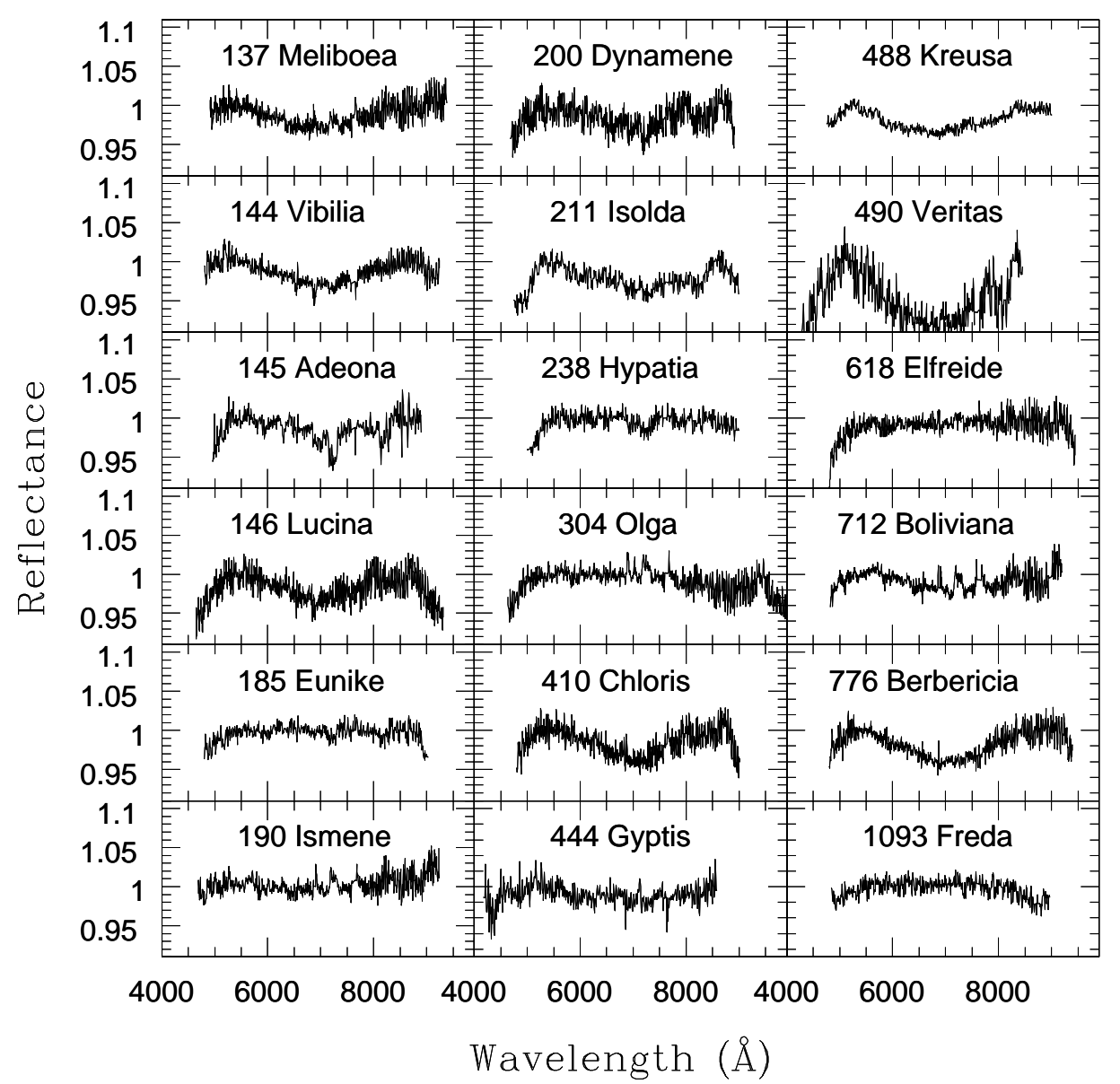

Fig. 3. Reflectance spectra of the observed asteroids. The spectra are normalized to $5500 \AA$ and a linear continuum has been removed

$\mathrm{F} \rightarrow \mathrm{B} \rightarrow \mathrm{C} \rightarrow \mathrm{G}$ asteroids (Vilas 1994). Two of the Gtype observed objects, 19 Fortuna and 130 Elektra show a well defined $0.7 \mu \mathrm{m}$ absorption band, while 1 Ceres has the $0.8-0.9 \mu \mathrm{m}$ band and two weak absorption bands at 0.6 and $0.67 \mu \mathrm{m}$, as observed by Vilas \& McFadden (1992) and Sawyer (1991).

Ceres has not the $0.7 \mu \mathrm{m}$ band, that seems to be a spectral characteristic of $G$ class objects, but, owing to its significant size, it cannot be considered typical of any asteroidal class.

\subsection{Comparison between CM2 chondrites and hydrated asteroids}

Finally, we have compared the spectra of the observed hydrated asteroids with those of several CM2 carbonaceous chondrite meteorites.

The spectra of the CM2 meteorites have been obtained from literature (Vilas et al. 1994) and have laboratory origin.

They reveal features probably due to aqueous altered materials on their surfaces. This investigation is important because the origin of meteorites is not well known yet.
Many factors affect the comparison between meteorites and asteroids (Pieters \& McFadden 1994):

- Physical preparation of a meteorite for measurement in the laboratory may not accurately reproduce the physical form of material on an asteroid surface.

- A significant limiting factor that affects the mineralogical interpretation of meteorite and asteroid spectra includes the signal to noise ratio (so the quality of the spectra), the spectral range of the measurement and the spectral resolution. In fact signal to noise ratio of dark asteroids spectra is generally quite lower than that of meteorites spectra obtained in the laboratory environment (100 or less for asteroids, about 3000 for meteorites). Available spectra of asteroids are limited by the sensitivity of detectors and the faintness of the signal from the object combined with the atmospheric and instrumental noise contribution.

- There are significant dimensional differences, so meteorites and asteroids may have experienced different thermal and chemical processes.

- Many asteroids have not contributed to our meteorite collection and may not correspond to any meteorite class. Moreover, asteroidal observations allow only the 
Table 2. Identification of absorption bands on the observed asteroids. For each band we indicate the central wavelength position, the depth and extension. We have identified only those bands whose depth is greater that the peak-to-peak scatter of the spectra due to noise. With the ? we have indicated those bands that seem to be present but are too weak with respect to the noise, so we believe that more observations are necessary to confirm them

\begin{tabular}{|c|c|c|c|c|}
\hline ASTEROID & $\begin{array}{c}\text { AQUEOUS } \\
\text { PRODUCTS }\end{array}$ & $\begin{array}{c}\text { OBSERVED } \\
\text { BANDS }(\mu \mathbf{m})\end{array}$ & DEPTH & EXTENSION \\
\hline 1 Ceres & $\mathrm{Y}$ & $\begin{array}{c}0.8-0.9 \\
0.60,0.67 ?\end{array}$ & $5.0 \%$ & $8000-9000 \AA$ \\
\hline 10 Hygiea & $?$ & $0.60 ?$ & & \\
\hline 19 Fortuna & Y & 0.70 & $5.0 \%$ & $5500-8500 \AA$ \\
\hline 24 Themis & $\mathrm{Y}$ & 0.70 & $4.0 \%$ & $5600-8200 \AA$ \\
\hline 34 Circe & $\mathrm{Y}$ & 0.70 & $2.3 \%$ & $5600-8300 \AA$ \\
\hline 38 Leda & $\mathrm{Y}$ & 0.70 & $3.4 \%$ & $5600-8300 \AA$ \\
\hline 41 Daphne & $\mathrm{Y}$ & 0.70 & $4.6 \%$ & $5400-8400 \AA$ \\
\hline 45 Eugenia & $\mathrm{N}$ & - & - & - \\
\hline 51 Nemausa & $\mathrm{Y}$ & 0.70 & $4.7 \%$ & $5600-8300 \AA$ \\
\hline 65 Cybele & $?$ & $0.60 ?$ & & \\
\hline 70 Panopaea & $\mathrm{Y}$ & 0.70 & $4 \%$ & $5600-8300 \AA$ \\
\hline 74 Galatea & $\mathrm{N}$ & - & - & - \\
\hline 104 Klymene & $\mathrm{Y}$ & $\begin{array}{c}0.70 \\
0.8-0.9 ?\end{array}$ & $2.5 \%$ & $5500-8200 \AA$ \\
\hline 105 Artemis & $\mathrm{Y}$ & 0.70 & $4.5 \%$ & $5700-8300 \AA$ \\
\hline 128 Nemesis & $\mathrm{N}$ & - & - & - \\
\hline 130 Elektra & $\mathrm{Y}$ & 0.70 & $4.7 \%$ & $5700-8400 \AA$ \\
\hline 137 Meliboea & $\mathrm{Y}$ & 0.70 & $3 \%$ & $5500-8200 \AA$ \\
\hline 144 Vibilia & $\mathrm{Y}$ & 0.70 & $3 \%$ & $5600-8300 \AA$ \\
\hline 145 Adeona & $\mathrm{Y}$ & 0.70 & $3.0 \%$ & $5600-8400 \AA$ \\
\hline 146 Lucina & $\mathrm{Y}$ & 0.70 & $3.4 \%$ & $5600-8000 \AA$ \\
\hline 185 Eunike & $\mathrm{N}$ & - & - & - \\
\hline 190 Ismene & $\mathrm{N}$ & - & - & - \\
\hline 200 Dynamene & $\mathrm{Y}$ & $\begin{array}{c}0.70 \\
0.8-0.9 ?\end{array}$ & $2.5 \%$ & $6000-8000 \AA$ \\
\hline 211 Isolda & $\mathrm{Y}$ & 0.70 & $3.4 \%$ & $5500-8500 \AA$ \\
\hline 238 Hypatia & $\mathrm{N}$ & - & - & - \\
\hline 304 Olga & $\mathrm{N}$ & - & - & - \\
\hline 410 Chloris & $\mathrm{Y}$ & 0.70 & $4.7 \%$ & $5500-8000 \AA$ \\
\hline 444 Gyptis & $\mathrm{Y}$ & $\begin{array}{c}0.70 \\
0.43 ? \\
0.50 ?\end{array}$ & $2.1 \%$ & $5600-8500 \AA$ \\
\hline 488 Kreusa & $\mathrm{Y}$ & 0.70 & $3.4 \%$ & $5400-8300 \AA$ \\
\hline 490 Veritas & $\mathrm{Y}$ & 0.70 & $6.9 \%$ & $5300-8300 \AA$ \\
\hline 618 Elfreide & $\mathrm{N}$ & - & - & - \\
\hline 712 Boliviana & $\mathrm{Y}$ & 0.70 & $3.1 \%$ & $5800-8300 \AA$ \\
\hline 776 Berbericia & $\mathrm{Y}$ & 0.70 & $4.5 \%$ & $5500-8300 \AA$ \\
\hline 1093 Freda & $\mathrm{N}$ & - & - & - \\
\hline
\end{tabular}

study of their surface properties, while meteorites may be derived also from the inner parts of the parent bodies.

- Space weathering influences the surface composition, and may affect asteroid and meteorites in different manner. This is a result of studies especially on lunar meteorites, and seems to produce lower albedo, weaker absorptions and a red-sloped continuum.

- Meteorites may be deeply altered from their pristine interspace condition by terrestrial weathering (Britt et al. 1992).
We compared our hydrated asteroids(those which have the $0.7 \mu \mathrm{m}$ band) with $7 \mathrm{CM} 2$ carbonaceous chondrites (see Fig. 6), whose spectra were treated as those of the asteroids, that is they were divided by a linear continuum defined by a linear least squares fit to the spectral data points.

We used the Chi-Square Fitting method to measure the agreement between each hydrated asteroid and the 7 CM2. The Chi-Square is defined in the following way:

$\chi^{2}=\sum_{i=1}^{N} \frac{\left(y a_{i}-y c_{i}\right)^{2}}{\sigma_{i}^{2}}$ 
Table 3. Values of the Chi-Square computed by the comparison of each hydrated asteroid with the 7 CM2 chondrites. The maximum likelihood between the asteroid and the meteorites is obtained for that CM2 which gives the least Chi-Square value (represented in boldface)

\begin{tabular}{|l|r|r|r|r|r|r|r|}
\hline ASTEROID & \multicolumn{7}{|c|}{ CM2 CARBONACEOUS CHONDRITES } \\
\hline & ALHA81002 & ALHA83100 & ALHA84029 & COLDBOKK & LEW90500 & MIGHEI & MURRAY \\
\hline 19 Fortuna & 190.37 & 136.69 & 210.48 & 149.68 & $\mathbf{1 1 7 . 5 6}$ & 129.17 & 153.68 \\
34 Circe & 568.79 & 390.56 & 675.35 & 402.86 & $\mathbf{1 9 9 . 6 3}$ & 287.78 & 266.88 \\
38 Leda & 338.15 & 249.55 & 428.34 & 248.07 & $\mathbf{1 1 2 . 9 6}$ & 161.63 & 176.49 \\
41 Daphne & 306.68 & 240.04 & 392.20 & 231.73 & $\mathbf{1 0 8 . 1 3}$ & 153.63 & 183.64 \\
51 Nemausa & 305.41 & 214.19 & 360.45 & 239.30 & $\mathbf{1 1 6 . 7 4}$ & 161.04 & 185.02 \\
70 Panopaea & 332.80 & 247.67 & 422.52 & 238.06 & $\mathbf{1 0 9 . 7 4}$ & 163.35 & 180.36 \\
104 Klymene & 472.69 & 331.95 & 581.13 & 342.68 & $\mathbf{1 6 0 . 4 8}$ & 227.91 & 215.54 \\
105 Artemis & 218.51 & 179.41 & 297.06 & 173.31 & $\mathbf{9 6 . 9 2}$ & 110.62 & 133.86 \\
130 Elektra & 353.08 & 227.35 & 430.03 & 234.53 & $\mathbf{8 9 . 7 5}$ & 152.77 & 152.00 \\
137 Meliboea & 438.91 & 312.04 & 539.44 & 318.57 & $\mathbf{1 4 4 . 9 3}$ & 213.06 & 212.33 \\
144 Vibilia & 399.74 & 275.89 & 491.40 & 278.69 & $\mathbf{1 1 7 . 7 6}$ & 184.28 & 187.58 \\
145 Adeona & 1155.17 & 1034.91 & 1250.80 & 1110.81 & $\mathbf{9 0 8 . 8 4}$ & 988.64 & 958.53 \\
146 Lucina & 406.56 & 284.63 & 493.35 & 289.16 & $\mathbf{1 3 6 . 2 5}$ & 198.52 & 201.60 \\
200 Dynamene & 519.33 & 396.66 & 609.94 & 431.76 & $\mathbf{2 5 1 . 6 9}$ & 320.57 & 313.02 \\
211 Isolda & 248.50 & 117.01 & 331.12 & 161.86 & $\mathbf{6 7 . 6 5}$ & 97.19 & 116.42 \\
410 Chloris & 404.02 & 273.46 & 484.06 & 299.08 & $\mathbf{1 2 8 . 1 0}$ & 197.68 & 201.90 \\
712 Boliviana & 541.99 & 373.99 & 647.68 & 378.72 & $\mathbf{1 9 6 . 3 5}$ & 266.76 & 243.87 \\
776 Berbericia & 300.72 & 218.19 & 378.16 & 222.01 & $\mathbf{9 4 . 8 9}$ & 145.71 & 164.82 \\
\hline
\end{tabular}

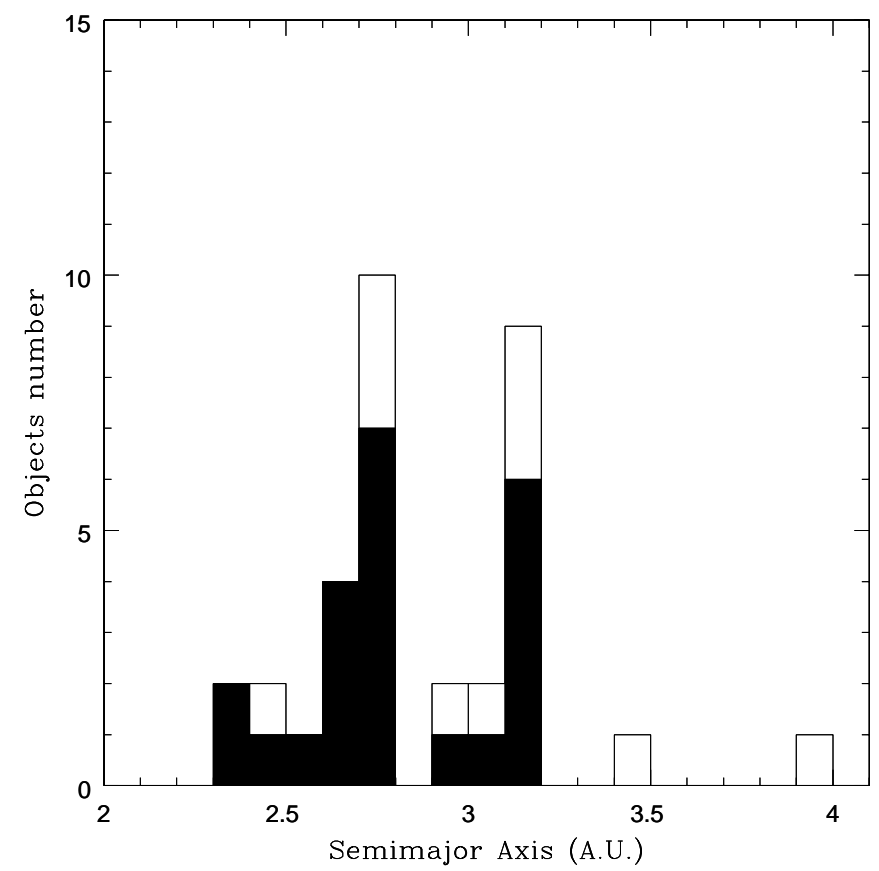

Fig. 4. Number of the observed objects as function of the semimajor axis. The black part represents the hydrated asteroids (only those which have clear and well identified hydration absorption features)

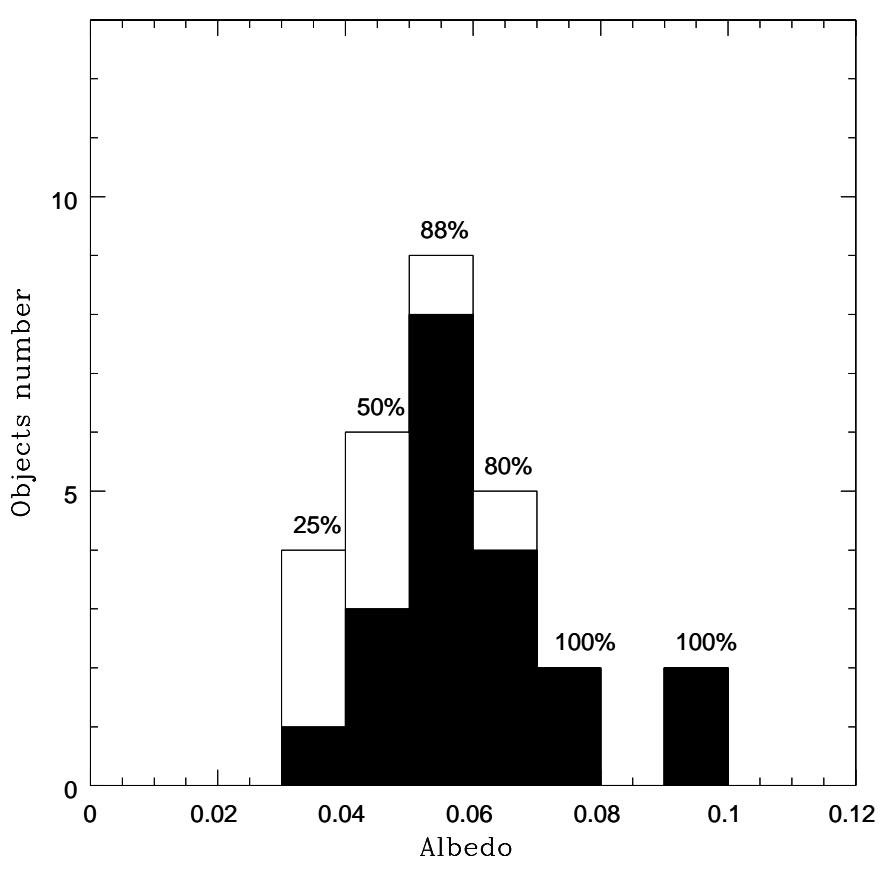

Fig. 5. Number of the observed objects as function of the geometric albedo. The black part represents the hydrated asteroids (only those which have clear and well identified hydration absorption features) 


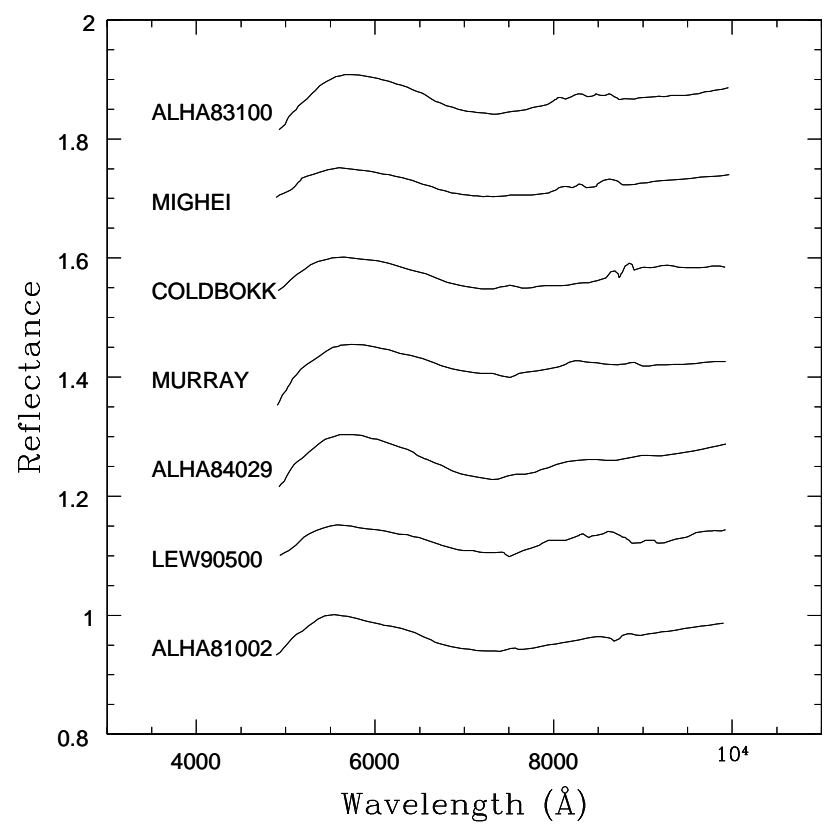

Fig. 6. Reflectance spectra of 7 CM2 carbonaceous chondrite meteorites (Vilas et al. 1993). The spectra were normalized to $5500 \AA$ and a linear continuum has been removed. They were offset by 0.15 in reflectance for clarity

where $\left(x_{i}, y a_{i}\right)$ are the mean data points $(x=$ wavelength; $y=$ reflectance) of the asteroid (i.e. a mean of the asteroidal signal, affected by noise); $\left(x_{i}, y c_{i}\right)$ are the data points of CM2 chondrites (these spectra were obtained in laboratory, so they have pratically no noise); $N=$ number of degree of freedom, i.e. the number of points in which we have divided the wavelength range; $\sigma_{i}$ is the standard deviation of the asteroidal data points.

We have computed the Chi-Square on 200 points $(N=$ 200).

As we have not a set of observations for each asteroid, but a single spectrum per asteroid, we have assumed $\sigma_{i}=$ costant $=0.02$, which is about the mean peak-topeak variation of the asteroidal signal.

The maximun likelihood estimate between each asteroid and the 7 "models" represented by the 7 CM2 meteorites is obtained when the Chi-Square assumes the least value.

The results of this quantitative comparison are summarized in Table 3 . The best meteoritical analog of all the hydrated asteroids is LEW90500 CM2: the other 6 CM2 have a deeper and wider $0.7 \mu \mathrm{m}$ absorption band than that of the asteroids.

In Figs. 7 and 8 we report some examples of the comparison between hydrated asteroids and LEW90500 CM2.

The differences in depth of the band and in its extension may depend on the degree of aqueous alteration

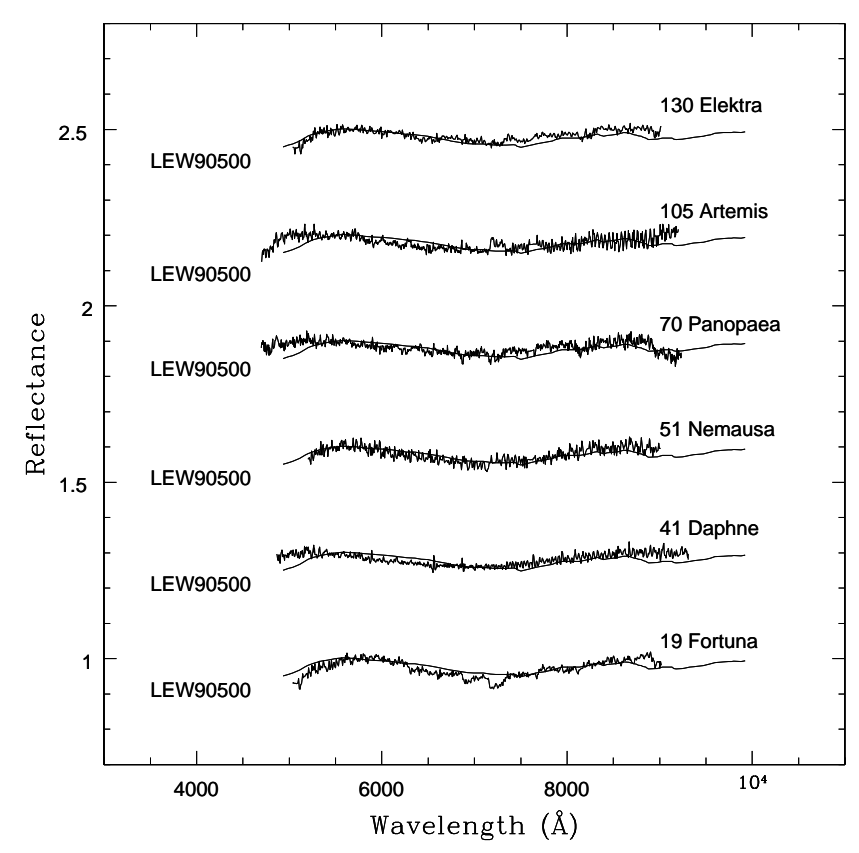

Fig. 7. Comparison between the asteroids 19 Fortuna, 41 Daphne, 51 Nemausa, 70 Panopaea, 105 Artemis and 130 Elektra with the carbonaceous chondrites LEW90500. The spectra are offset by 0.3 in reflectance for clarity

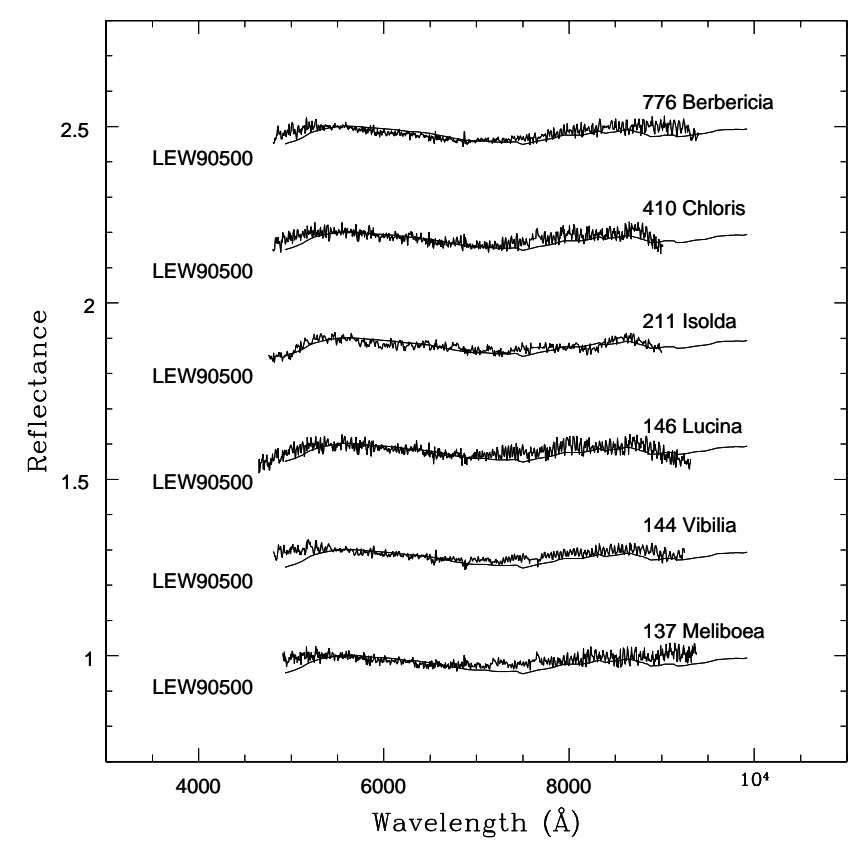

Fig. 8. Comparison between the asteroids 137 Meliboea, 144 Vibilia, 146 Lucina, 211 Isolda, 410 Chloris and 776 Berbericia with the carbonaceous chondrites LEW90500. The spectra are offset by 0.3 in reflectance for clarity 
and on the presence of different amount of $\mathrm{Fe}$ in silicates crystal lattices. Moreover, laboratory experiments show that the reflectance of a mineral mixture is nonlinear and is a function of viewing geometry and properties of the particles such as single scattering albedo (efficiency of an average particle to scatter and not absorb light), porosity, diameters and mass fractions (Burbine et al. 1996). So small differences in composition or in particle sizes and properties are sufficient to produce a different spectral response.

The good match between several observed hydrated asteroids and CM2 meteorites, in particular LEW90500, resulting from our analysis, is a valid confirmation that aqueous altered asteroids could be the parents of CM2 carbonaceous chondrite meteorites.

\section{Conclusions}

We obtained visible spectra of 34 asteroids belonging to C, $\mathrm{G}$, and $\mathrm{P}$ classes, localized between 2.2 and 3.9 AU. Our analysis reveals that more than $65 \%$ of the investigated asteroids show the presence of absorption bands due to aqueous alteration products $(0.6,0.7,0.8-0.9 \mu \mathrm{m}$, depth between $2 \%$ and $6 \%$ with respect to the continuum), in particular of the $0.7 \mu \mathrm{m}$ band, which is the most characteristic feature of the hydrated materials in the visible. Our data also confirm the existence of a relationship between the albedo of the objects and the aqueous alteration process: in fact the percentage of the observed hydrated asteroids grows as albedo increases. We also found hydration features on 4 asteroids located outside the "aqueous alteration zone" as defined by Vilas (1994). Our results confirm those recently obtained by Barucci et al. (1998).

We compared the spectra of the observed hydrated asteroids with those of 7 CM2 carbonaceous chondrite meteorites. This comparison reveals a good match (differences $\leq 3 \%$ ) between the meteorites and most of the observed objects, suggesting that hydrated asteroids might be the parent bodies of CM2 meteorites.

\section{References}

Barucci M.A., Fulchignoni M., Lazzarin M., 1996, Planet. Space Sci. 44, 1047

Barucci M.A., Doressoundiram A., Fulchignoni M., Lazzarin M., Florczak M., Angeli C., Lazzaro D., 1998, Icarus 132, 388

Britt D.T., Tholen D.J., Bell J.F., Pieters C.M., 1992, Icarus 99, 153

Burbine T.H., Meibon A., Binzel R.P., 1996, Meteor. Planet. Sci. 31,607

Gradie J.C., Tedesco E.F., 1982, Sci 216, 1405

Hardorp J., 1978, A\&A 63, 383

Herbert F., 1989, Icarus 78, 402

Jones T.D., Lebofsky L., Lewis J., Marley M., 1990, Icarus 88, 172

King T.V.V., Clarck R.N., Calvin W.M., Sherman D.M., Brown R.H., 1992, Sci 255, 1551

Lazzarin M., Barbieri C., Barucci M.A., 1995, AJ 110, 3058

Lebofsky L.A., 1978, MNRAS 182, 17

Lebofsky L.A., 1980, AJ 85, 573

Lebofsky L.A., Feierberg M.A., Tokunaga A.T., Larson H.P., Johnson J.R., 1981, Icarus 48, 453

Lebofsky L.A., Jones T., Owensby P.D., Feierberg M.A., Consolmagno G.J., 1990, Icarus 83, 16

Pieters C.M., McFadden L.A., 1994, Annu. Rev. Earth Planet. Sci. 22,457

Sawyer S.R., 1991, PhD thesis, The University of Texas at Austin, A hight resolution CCD Spectroscopic Survey of Low Albedo Main Belt Asteroids

Shimazu H., Teresawa T., 1995, Jou. Geoph. Res. 100, 16923

Vilas F., Smith B.A., 1985, Icarus 64, 503

Vilas F., McFadden L.A., 1992, Icarus 100, 85

Vilas F., Hatch E.C., Larson S.M., Sawyer S.R., Gaffey M.J., 1993, Icarus 102, 225

Vilas F., Larson S.M., Hatch E.C., Jarvis K.S., 1993b, Icarus 105,67

Vilas F., 1994, Icarus 111, 456

Vilas F., Jarvis K.S., Gaffey M.J., 1994, Icarus 109, 274

Zolensky M.E., McSween M., 1988, in: Kerridge J.F., Matthews M.S. (eds.) Meteorites and the early Solar System. Univ. of Arizona Press, Tucson, p. 114 